\begin{tabular}{|c|c|}
\hline Title & Radiative cooling for continuous thermoelectric power generation in day and night \\
\hline Author(s) & Ishii, Satoshi; Dao, Thang Duy; Nagao, Tadaaki \\
\hline Citation & $\begin{array}{l}\text { A pplied physics letters, 117, } 013901 \\
\text { https://doi.org/10.1063/5.0010190 }\end{array}$ \\
\hline Issue Date & $2020-07-06$ \\
\hline Doc URL & http:/hdl.handle.net/2115/82136 \\
\hline Rights & $\begin{array}{l}\text { This article may be downloaded for personal use only. Any other use requires prior permission of the author and A IP } \\
\text { Publishing. This article appeared in A pplied Physics Letters } 117 \text { (2020) and may be found at } \\
\text { https://aip.scitation.org/doi } / 10.106355 .0010190 \text {. }\end{array}$ \\
\hline Type & article \\
\hline File Information & A ppl. Phys. Lett. 117_013901.pdf \\
\hline
\end{tabular}

Instructions for use 


\section{Radiative cooling for continuous thermoelectric power generation in day and night}

Cite as: Appl. Phys. Lett. 117, 013901 (2020); https://doi.org/10.1063/5.0010190

Submitted: 08 April 2020 . Accepted: 04 June 2020 . Published Online: 07 July 2020

Satoshi Ishii (D), Thang Duy Dao (D), and Tadaaki Nagao (iD)

\section{COLLECTIONS}

F This paper was selected as Featured

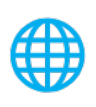

View Online

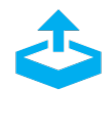

Export Citation

\section{ARTICLES YOU MAY BE INTERESTED IN}

Widegap $\mathrm{CH}_{3} \mathrm{NH}_{3} \mathrm{PbBr}_{3}$ solar cells for optical wireless power transmission application Applied Physics Letters 117, 013902 (2020); https://doi.org/10.1063/5.0010009

Room-temperature operation of c-plane GaN vertical cavity surface emitting laser on conductive nanoporous distributed Bragg reflector

Applied Physics Letters 117, 011101 (2020); https://doi.org/10.1063/5.0012281

Low-frequency Raman scattering in $\mathrm{WSe}_{2}-\mathrm{MoSe}_{2}$ heterobilayers: Evidence for atomic reconstruction

Applied Physics Letters 117, 013104 (2020); https://doi.org/10.1063/5.0012249

\section{Lock-in Amplifiers up to $600 \mathrm{MHz}$}
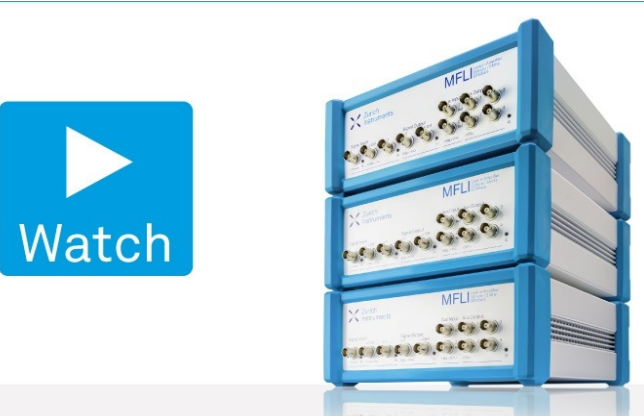


\title{
Radiative cooling for continuous thermoelectric power generation in day and night $\odot$
}

\author{
Cite as: Appl. Phys. Lett. 117, 013901 (2020); doi: 10.1063/5.0010190 \\ Submitted: 8 April 2020 - Accepted: 4 June 2020 • \\ Published Online: 7 July 2020

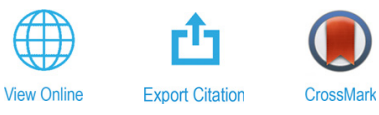

Satoshi Ishii, ${ }^{1,2,3, a)}$ (D) Thang Duy Dao, (D) and Tadaaki Nagao ${ }^{1,4}$ (D)

\begin{abstract}
AFFILIATIONS
'International Center for Materials Nanoarchitectonics (MANA), National Institute for Materials Science (NIMS), Tsukuba, Ibaraki 305-0044, Japan

${ }^{2}$ School of Pure and Applied Sciences, University of Tsukuba, Tsukuba, Ibaraki 305-8577, Japan

${ }^{3}$ Japan Science and Technology Agency, PRESTO, 4-1-8 Honcho, Kawaguchi, Saitama 332-0012, Japan

${ }^{4}$ Department of Condensed Matter Physics, Graduate School of Science, Hokkaido University, Kita-10 Nishi-8 Kita-ku, Sapporo 060-0810, Japan
\end{abstract}

${ }^{a)}$ Author to whom correspondence should be addressed: sishii@nims.go.jp

\begin{abstract}
Harvesting energy from the environment often suffers from discontinuity in power generation. Typical examples of technologies where this problem occurs are photovoltaics and solar heat power generation, which can only generate power during the day. In this study, the temperature difference caused by radiative cooling is utilized to generate thermoelectric power continuously throughout the day. The emitter has a wavelength-selective emissivity such that it is radiatively cooled all the time and its surface temperature is always cooler than the bottom side of the thermoelectric module which is placed below the selective emitter. The device placed outdoor generates thermoelectric voltage during the day and night without dropping to zero. The device can be used as a power supply for sensors and monitors placed outdoors.
\end{abstract}

Published under license by AIP Publishing. https://doi.org/10.1063/5.0010190

Increasing numbers of sensors are being used in industry and daily life for various purposes. The number of sensors is estimated to be on the order of trillions within a few years. While some sensors are integrated into hardware or buildings that are powered by the electricity network, others are expected to work in off-grid situations, such as monitoring outdoor temperature or traffic volume. Although there has been much effort devoted to develop energy-efficient sensors and electric circuits, minimum electric power should be supplied to those sensors. Batteries can be used to power off-grid sensors or monitors; however, they need to be recharged regularly, and using batteries increases the cost of installation.

In this context, energy harvesting from the environment is critical to realize self-powered sensors. Energy-harvesting techniques generate electricity from various sources, including light, heat, vibration, and electromagnetic waves. For outdoor usage, sunlight is a universal energy source. Photovoltaic cells are certainly the choice for generating electricity from sunlight. Thermoelectric (TE) devices can also be powered by sunlight in the form of solar heat. By using broadband or infrared (IR) absorbers, TE devices can potentially utilize the IR portion of sunlight that is typically unused in photovoltaic cells. Solarpowered TE generators were patented more than a hundred years ago, ${ }^{1,2}$ and primitive experiments have been carried out since the early 20th century. ${ }^{3-5}$ More recent studies combined TE devices with various solar concentrators, heat pipes, ${ }^{6}$ and sophisticated thermal management. ${ }^{7}$ Although these studies have improved TE conversion efficiency, TE generators relying on solar heat generate electricity only in the daytime, similar to photovoltaic cells.

To generate TE power at night, there have been attempts to take advantage of radiative cooling. Instead of a TE device being heated by the sun, a TE device is cooled by a thermal emitter placed on top of the TE device where heat is radiatively transferred to space. A thermal emitter can be a broadband emitter (BE) having high emissivity in the entire mid-IR (MIR) spectrum. A much more effective emitter has high emissivity only in the atmospheric window $(8-13 \mu \mathrm{m})$ to avoid the absorption from the atmosphere.

Nighttime TE power generation has been demonstrated previously. 8 Actually, Ref. 8 has shown that a thermal emitter acts as a solar absorber such that the system can generate TE power in both nighttime and daytime. However, the sign of the TE voltage changes from night to day and vice versa. More importantly, the TE voltage becomes zero when the sign changes. This can be a disadvantage, for instance, if one wants to power sensors for $24 \mathrm{~h}$. 
To avoid the TE voltage dropping to zero, the thermal emitter placed on a TE device can be replaced by a wavelength-selective emitter (SE) that radiatively cools day and night. An SE has to meet the following spectral-dependent conditions. For emissivity, it must be nearly zero in solar spectrum and close to unity in the atmospheric window. For reflectivity, it needs to be close to unity in the solar spectrum and nearly zero in the atmospheric window. ${ }^{10-12}$ Thus, an SE can cool to sub-ambient temperature even in the daytime without being heated by sunlight. Efforts have been made for decades to achieve daytime radiative cooling. ${ }^{13,14}$ Recently, daytime radiative cooling has attracted significant attention because of growing concerns about global warming and a sustainable society. ${ }^{15}$ Intensive research on daytime radiative coolers has advanced their performances by developing different kinds of nanophotonic structures ${ }^{16-23}$ and by suppressing heat losses. ${ }^{21,24}$ Application-oriented research has been conducted, such as studies on cooling buildings ${ }^{25}$ and human skin, ${ }^{26}$ and visible-colored coolers have even been developed. ${ }^{2}$

In the current work, continuous TE generation without dropping to zero was demonstrated outdoors by attaching an SE on a TE device. The SE results from a glass substrate and an aluminum (Al) thin film. This is simpler than other sample structures. From the generated TE voltage, the maximum temperature difference between the top and bottom of the TE device was estimated to be $\sim 5^{\circ} \mathrm{C}$ in daytime without using a vacuum chamber or a cover to minimize convection heat losses. As a control sample, a BE, which was a blackbody paint-coated glass substrate, was also prepared and attached to a TE device. The control sample placed outdoor generated opposite TE voltages in
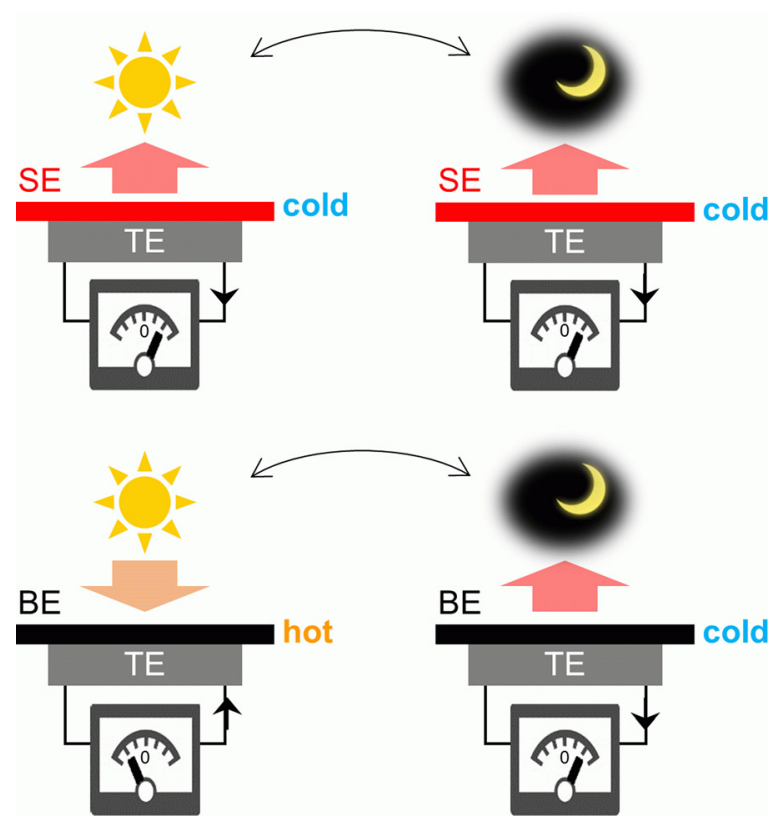

FIG. 1. Conceptual drawing of thermoelectric (TE) devices having a wavelengthselective emitter (SE) and a broadband emitter (BE) on the top. When an SE is on the top, the top temperature is always cooler than the bottom, and the sign of the TE voltage does not change; however, when a BE is on the top, the top is hotter and cooler than the bottom in daytime and nighttime, respectively. Thus, the generated TE voltage changes its sign. daytime and nighttime. This is similar to the results reported previously. ${ }^{8}$ To confirm the results recorded outdoor, indoor experiments were performed using a solar simulator and a Peltier module, and they confirmed the results of outdoor experiments. The results highlight the advantage of a TE device assisted with an SE for continuous power generation without downtime. This approach is ideally suited for continuous outdoor sensing or monitoring without batteries.

A schematic of the sample design for a continuous TE generator is shown in Fig. 1. An SE, which acts as a daytime radiative cooler, is placed on a TE device. Because the SE is radiatively cooled all the time, the top side of the TE device is always cooler than its bottom; hence, continuous TE generation is maintained. In contrast, if a BE is instead placed on a TE device, the $\mathrm{BE}$ is radiatively cooled during nighttime but is heated by sunlight during daytime. Thus, as time shifts from night to morning, the top temperature gradually increases, and the temperature difference between the top and bottom becomes zero. Something similar happens when time passes from day to evening. On those occasions, the TE generation becomes zero. In addition, as described later, sudden weather changes (such as the appearance of clouds) also reduce the temperature difference to zero.

The designs and photos of the SE and BE are shown in Figs. 2(a) and 2(b). The SE has a 100-nm-thick Al film on the back of a glass substrate (labeled "glass/Al"). The BE has a blackbody paint on top of the glass substrate (labeled "blackbody"). Both samples had TE devices on the bottom. Figure 2(c) shows the thermography of the samples placed on the roof in the morning. Even though the glass/Al sample has a simple design, it is cooler than the roof because of the radiative heat transfer from the sample to the outer space. On the contrary, the temperature of the blackbody sample is higher than that of the roof. This is because the blackbody paint absorbed more sunlight than the rooftop. A thermography image was also taken in the laboratory, as

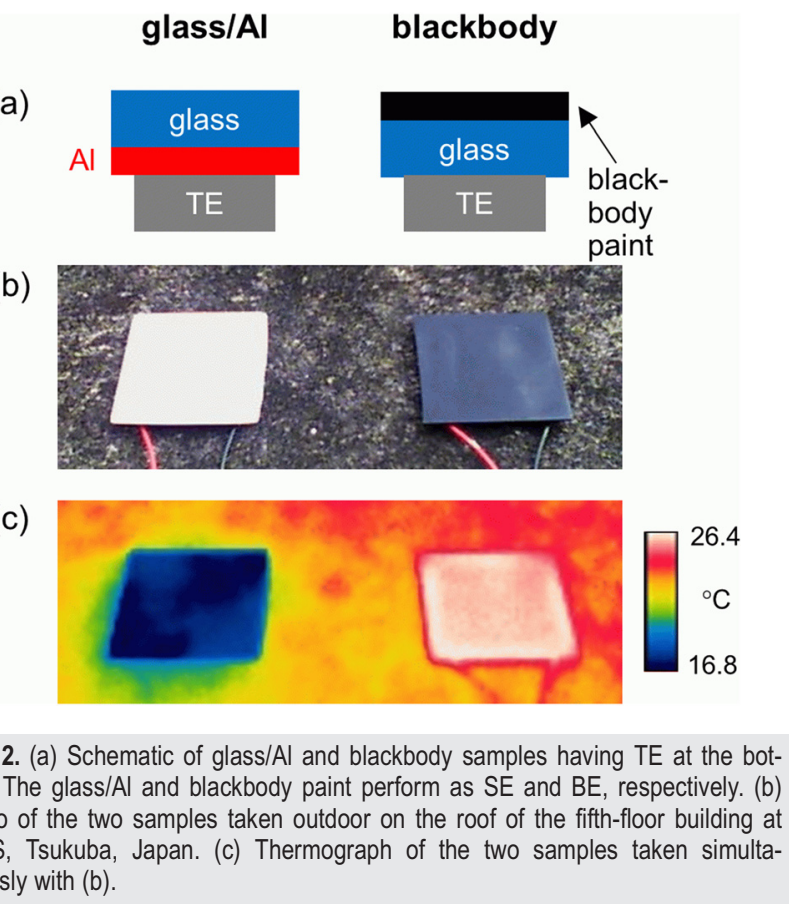


shown in Fig. S1. Because radiative heat transfer to space was not possible in the laboratory, the SE sample was not cooled, which in turn indicates that the cooling effect shown in Fig. 2(c) was caused by radiative cooling. The BE sample in the laboratory was also at room temperature because it was not heated by sunlight.

The thermography image can be understood better by referring to the optical properties of the sample. Figures 3(a) and 3(b) show the specular reflectivity and emissivity, respectively, of the sample from $\mathrm{UV}$ to MIR range. The glass/Al sample has high reflectivity in the optical range because of the $\mathrm{Al}$ film below the glass. In the MIR range, the glass is nearly a blackbody, so that the emissivity is close to unity. Because of these optical properties, the glass/Al sample can be cooled by radiative heat transfer in daytime and nighttime. However, the blackbody sample had high emissivity across the entire spectrum, as expected. Therefore, it became heated during daytime by sunlight and radiatively cooled during nighttime.

After the basic characterization of the sample, the TE voltages measured outdoor of the two samples were plotted, as shown in Fig. 3(c). Solar irradiance, which was measured near the samples at the same time, is shown in Fig. 3(d). Temperature and humidity are plotted in Fig. S2. As expected, the glass/Al sample generated a TE voltage continuously without dropping to zero. The result indicates that the top TE temperature was always cooler than the bottom. However, the TE voltage of the blackbody sample crossed zero when the time passed from night to day and day to night, which indicates that the top temperature of the TE became hotter than the bottom in the morning and vice versa in the early evening. Interestingly, there were occasions when the TE voltage changed its sign during the day. The timing matches to the sudden decrease in the solar irradiance. Because the heat capacitance of the sample was small, the sudden decrease in the solar irradiance resulted in a lowering of the top temperature of the blackbody sample. Because the roof had a much larger heat capacitance than the sample, the temperature change with respect to solar irradiance was slower than that of the sample, so that the sudden decrease in the solar irradiance changed the sign of the temperature difference.
During the day, the magnitude of the TE voltage for the blackbody sample was larger than that of the glass/ $\mathrm{Al}$ sample before around $12 \mathrm{pm}$. The reason is that solar irradiance was higher than the thermal radiation from the sample to space. However, this relation changes if one heats the sample back, as in the later discussion of indoor measurement. Another thing to be appreciated is that from 1 to $5 \mathrm{pm}$, it was cloudy, which can be inferred from the lower solar irradiance. During this period, the TE voltage of the glass/Al sample is larger than that of the blackbody sample. This happened due to the lowering of the solar irradiance by the cloud. In contrast, the glass/Al sample generated nearly constant TE voltage regardless of the weather change. Insensitiveness to the weather is another advantage of the glass/TE sample. During nighttime, the TE voltages of the two samples nearly overlapped. This is because the glass substrate behaved nearly as a blackbody in the MIR, as shown in Fig. 3(a), so that both samples were similarly cooled by radiative heat transfer.

In Fig. 3(c), the temperature differences of the top and bottom surfaces of the two samples are also plotted in the right axis. The temperature differences were deduced from the TE voltage, where the temperature difference to the TE voltage ratio was measured separately in the laboratory (see the supplementary material). Figure 3(c) shows that the maximum temperature differences of the glass/Al and blackbody samples during the day were +5 and $-15 \mathrm{~K}$, respectively. At night, the temperature differences were approximately $2-4 \mathrm{~K}$ for both samples. A heat transfer calculation was carried out for the glass/Al sample, which verified the experiment (see the supplementary material).

To confirm the measurements outdoor, indoor experiments were carried out as well. First, TE voltage generation was measured by placing a sample facing toward a blackbody-painted Peltier module, as shown in Fig. S3. Radiative cooling and heating resulted in positive and negative TE voltages, respectively, for the two samples. The results confirm that radiative cooling and heating generated a temperature difference between the top and bottom of the samples.

Then, three conditions were tested in the laboratory, as schematically shown in Fig. 4(a). A blackbody-painted Peltier module and a (a)

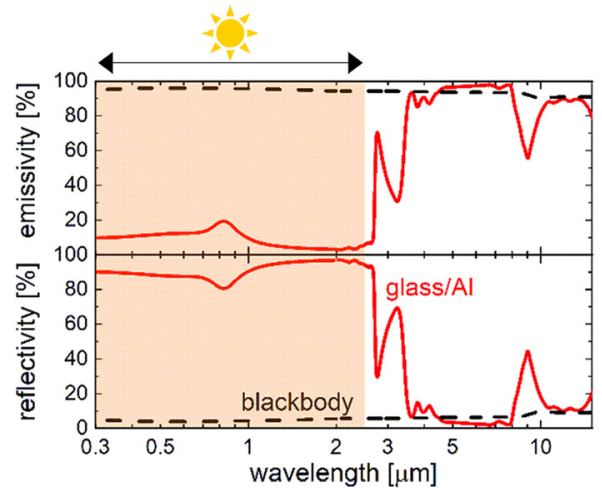

(c)

(d)

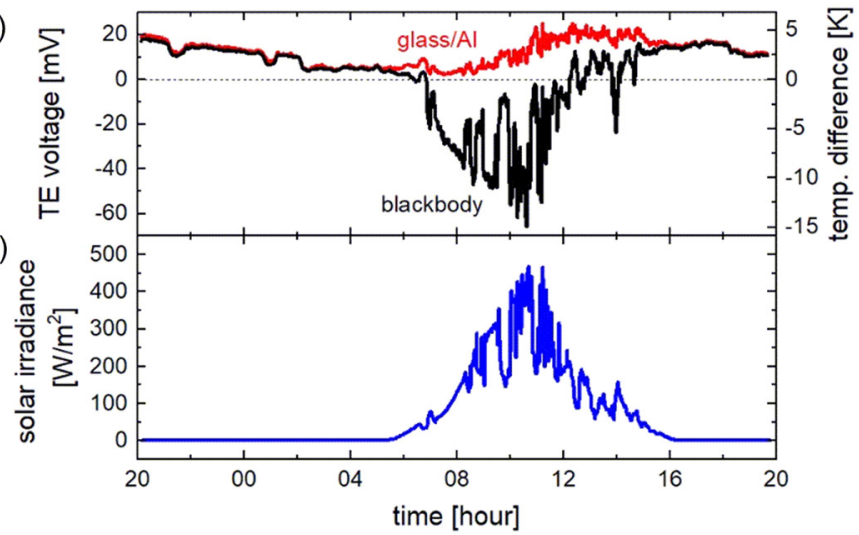

FIG. 3. (a) Emissivity and (b) reflectivity of glass/Al and blackbody samples measured from UV to MIR. (c) TE voltages of glass/Al and blackbody samples measured outdoor. The left axis and right axis show the raw TE voltage and the temperature difference between the top and bottom of the samples. The temperature difference shown in the right axis was converted from the measured TE voltage shown in the left axis. (d) Solar irradiance measured together with the TE voltage. The sensor was placed next to the samples. 
(a)

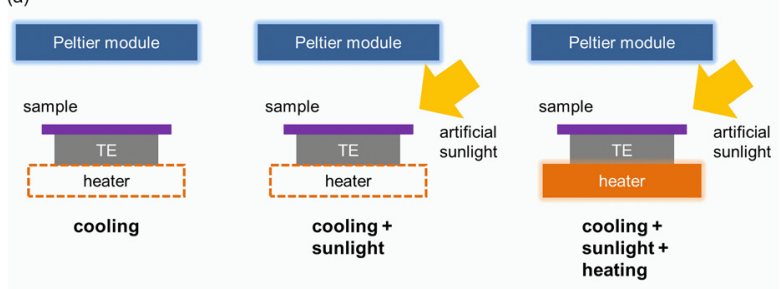

(b)

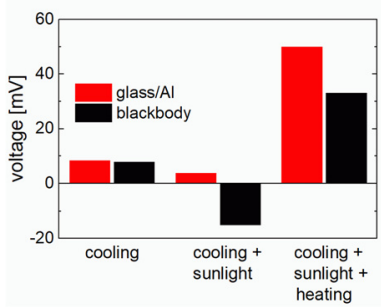

(c)

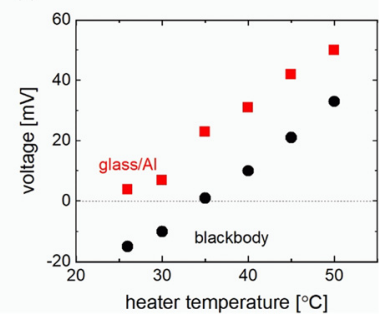

FIG. 4. (a) Schematic of the three different conditions tested in the laboratory. The labels cooling, sunlight, and heater correspond to radiative cooling by a Peltier module, sunlight irradiation by a solar simulator, and conductive heating by a heating stage, respectively. (b) Comparison of TE voltage generated at three different conditions described in (a). (c) TE voltage plotted against the heater temperature with the setting labeled as cooling + sunlight + heating in (a)

solar simulator were used to imitate the universe and the sun, respectively. In Fig. 4(a), "cooling," "sunlight," and "heater" refer to radiative cooling by a Peltier module, irradiation by a solar simulator, and conductive heating by a heating stage, respectively. The settings shown as "cooling" and "cooling + sunlight" ("cooling + sunlight + heater") correspond to nighttime and daytime, respectively.

In Fig. 4(b), the performances of the three conditions are compared. When there is only radiative cooling, the two samples generate similar TE voltages, which is consistent with the outdoor experiment at night, as shown in Fig. 3(c). When there are sunlight and radiative cooling, the glass/Al and blackbody samples generate positive and negative TE voltages, respectively. The magnitude of the TE voltage was larger for the blackbody sample because the solar irradiance was larger than the emissive heat flux. Another factor that made the TE voltage of the glass/Al sample smaller than that of the blackbody sample was the sunlight absorption of $\mathrm{Al}$ film, which was estimated to be $10 \%$ of the total solar irradiance. Solar heating cancels radiative cooling to make the temperature difference smaller. The situation changed drastically when the samples were heated from the back. The glass/Al sample generated a higher TE voltage than the blackbody sample. As discussed earlier, for the glass/Al sample, radiative cooling at the top and heating from the bottom increased the temperature difference between the top and bottom. In contrast, the temperature difference between the top and bottom became smaller for the blackbody sample.

To provide better insight into the backside heating, the heater temperature dependence of the TE voltage is plotted in Fig. 4(c). The $\mathrm{TE}$ voltage of the glass/Al sample monotonically increased from 3.8 to $50 \mathrm{mV}$ when the temperature increased from room temperature to $50^{\circ} \mathrm{C}$. In this case, radiative cooling at the top and conductive heating at the bottom resulted in increasing the temperature difference between the top and bottom. The blackbody sample also demonstrated a monotonic TE voltage increase from -15 to $+33 \mathrm{mV}$; however, the
TE voltage of the blackbody sample was always lower than that of the glass/Al sample. The reason is that the temperature difference generated by the heater from the bottom was partially canceled by solar heat at the top. Overall, the indoor experiment elucidated that the glass/Al sample can efficiently take advantage of the heat from the back. In a real situation, the heater can be waste heat from a building or a heat exchanger. Our study indicates that an SE combined with a TE module can simultaneously harvest radiative cooling and waste heat to enhance the TE generation. Because an SE combined with a TE module can continuously generate TE power, it is potentially useful to selfpower monitors or sensors placed outdoor.

To conclude, an SE, which can radiatively cool throughout the day mounted on a TE module, has been shown to generate TE voltage during the day and night continuously without dropping to zero. The structure was made of a glass substrate and an $\mathrm{Al}$ thin film. The continuous TE voltage was possible because the top side of the TE module was always cooled by the radiative heat transfer to space, and there was always a temperature difference in the same direction. In contrast, when a blackbody-painted glass substrate was mounted on a TE module, the TE voltage had opposite signs in the daytime and nighttime, and there were occasions when the TE voltage was zero. Among outdoor energy-harvesting techniques, it is challenging to generate power all day without using batteries. Thus, a daytime radiative-cooling structure combined with a TE module can be a promising strategy to supply power for sensors and monitors placed outdoors.

See the supplementary material for the experimental method, heat transfer analysis, the thermography taken indoor, the temperature and humidity data, and the thermoelectric voltage by indoor radiative heat transfer.

This work was supported by JST, PRESTO Grant No. JPMJPR19I2, Japan, and JSPS KAKENHI Grant Nos. 16H06364, 17K19045, and 17H04801, Japan.

\section{DATA AVAILABILITY}

The data that support the findings of this study are available within the article and its supplementary material.

\section{REFERENCES}

${ }^{1}$ E. Weston, U.S. patent 389,124 (1888).

${ }^{2}$ W. W. Coblentz, U.S. patent 1,077,219 (1913).

${ }^{3}$ P. Kirkpatrick, Sci. Am. 122, 677 (1920).

${ }^{4}$ W. W. Coblentz, Sci. Papers Bur. Stand. 19, 375 (1923).

${ }^{5}$ M. Telkes, J. Appl. Phys. 25(6), 765 (1954).

${ }^{6}$ W. He, Y. Su, Y. Q. Wang, S. B. Riffat, and J. Ji, Renewable Energy 37(1), 142 (2012).

${ }^{7}$ D. Kraemer, B. Poudel, H.-P. Feng, J. C. Caylor, B. Yu, X. Yan, Y. Ma, X. Wang, D. Wang, A. Muto, K. McEnaney, M. Chiesa, Z. Ren, and G. Chen, Nat. Mater. 10(7), 532 (2011).

${ }^{8}$ A. Yamada, Japan patent JPB 002716861 (1997).

${ }^{9}$ A. P. Raman, W. Li, and S. Fan, Joule 3, 2679 (2019).

${ }^{10}$ X. Sun, Y. Sun, Z. Zhou, M. Ashraful Alam, and P. Bermel, Nanophotonics 6(5), 997 (2017).

${ }^{11}$ M. Zeyghami, D. Y. Goswami, and E. Stefanakos, Sol. Energy Mater. Sol, Cells 178, 115 (2018).

${ }^{12}$ D. Zhao, A. Aili, Y. Zhai, S. Xu, G. Tan, X. Yin, and R. Yang, Appl. Phys. Rev. 6(2), 021306 (2019).

${ }^{13}$ S. Catalanotti, V. Cuomo, G. Piro, D. Ruggi, V. Silvestrini, and G. Troise, Sol. Energy 17(2), 83 (1975) 
${ }^{14}$ T. M. J. Nilsson, G. A. Niklasson, and C. G. Granqvist, Sol. Energy Mater. Sol. Cells 28(2), 175 (1992).

${ }^{15}$ A. P. Raman, M. A. Anoma, L. Zhu, E. Rephaeli, and S. Fan, Nature 515, 540 (2014).

${ }^{16}$ J.-L. Kou, Z. Jurado, Z. Chen, S. Fan, and A. J. Minnich, ACS Photonics 4(3), 626 (2017).

${ }^{17}$ Y. Zhai, Y. Ma, S. N. David, D. Zhao, R. Lou, G. Tan, R. Yang, and X. Yin, Science 355(6329), 1062 (2017).

${ }^{18}$ J. Mandal, Y. Fu, A. C. Overvig, M. Jia, K. Sun, N. N. Shi, H. Zhou, X. Xiao, N. Yu, and Y. Yang, Science 362(6412), 315 (2018).

${ }^{19}$ B. Bhatia, A. Leroy, Y. Shen, L. Zhao, M. Gianello, D. Li, T. Gu, J. Hu, M. Soljačić, and E. N. Wang, Nat. Commun. 9(1), 5001 (2018).

${ }^{20}$ T. Suichi, A. Ishikawa, Y. Hayashi, and K. Tsuruta, AIP Adv. 8(5), 055124 (2018).
${ }^{21}$ A. Leroy, B. Bhatia, C. C. Kelsall, A. Castillejo-Cuberos, M. Di Capua H, L. Zhao, L. Zhang, A. M. Guzman, and E. N. Wang, Sci. Adv. 5(10), eaat 9480 (2019).

${ }^{22} \mathrm{~T}$. Li, Y. Zhai, S. He, W. Gan, Z. Wei, M. Heidarinejad, D. Dalgo, R. Mi, X. Zhao, J. Song, J. Dai, C. Chen, A. Aili, A. Vellore, A. Martini, R. Yang, J. Srebric, X. Yin, and L. Hu, Science 364(6442), 760 (2019).

${ }^{23}$ Y. Fu, J. Yang, Y. S. Su, W. Du, and Y. G. Ma, Sol. Energy Mater. Sol. Cells 191, 50 (2019).

${ }^{24}$ Z. Chen, L. Zhu, A. Raman, and S. Fan, Nat. Commun. 7(1), 13729 (2016).

${ }^{25}$ E. A. Goldstein, A. P. Raman, and S. Fan, Nat. Energy 2, 17143 (2017).

${ }^{26}$ P.-C. Hsu, A. Y. Song, P. B. Catrysse, C. Liu, Y. Peng, J. Xie, S. Fan, and Y. Cui, Science 353(6303), 1019 (2016).

${ }^{27}$ G. J. Lee, Y. J. Kim, H. M. Kim, Y. J. Yoo, and Y. M. Song, Adv. Opt. Mater. 6(22), 1800707 (2018). 